\title{
In-Orbit Radiometric Calibration of the FORMOSAT-2 Remote Sensing Instrument
}

\author{
Tang-Huang Lin $^{1,3}$ and Gin-Rong Liu ${ }^{1,2, *}$ \\ ${ }^{1}$ Center for Space and Remote Sensing Research, National Central University, Jhongli 320, Taiwan, ROC \\ ${ }^{2}$ Institute of Atmospheric Science, National Central University, Jhongli 320, Taiwan, ROC \\ ${ }^{3}$ Institute of Space Science, National Central University, Jhongli 320, Taiwan, ROC
}

Received 6 June 2008, accepted 31 December 2008

\begin{abstract}
This principle focus of this study is the absolute radiometric calibrations of FORMOSAT-2 RSI imagery in orbit. There are two principal parts for achieving this calibration. The first is the assessment of the calibration site by examining atmospheric observations from ground stations and field measurements via ground-based radiometric instruments. After careful consideration based on the essential requirements for a suitable calibration site i.e., prevailing clear and clean atmosphere conditions over a wide, flat and near lambertian surface with high reflectance, the airport on Dongsha Island was considered to be an suitable site. The next phase is to design a scheme for the field campaign at the calibration site for radiometric calibration. Thus a synchronous experiment acquiring simultaneous measurements from the FORMOSAT-2 Remote Sensing Instrument (RSI) sensor and ground-based instruments was proposed and implemented for the period 16 to 19 September 2004. As a result, a set of reasonable radiometric coefficients for the absolute radiance calibration of the RSI was successfully constructed via the radiative transfer code associated with the synchronous measurements in this study.
\end{abstract}

Key words: FORMOSAT-2 RSI, Radiometric calibration, In-orbit

Citation: Lin, T. H. and G. R. Liu, 2009: In-orbit radiometric calibration of the FORMOSAT-2 remote sensing instrument. Terr. Atmos. Ocean. Sci., 20, 833-838, doi: 10.3319/TAO.2008.12.31.01(A)

\section{INTRODUCTION}

The FORMOSAT-2 satellite was launched successfully on 21 May 2004. Its high spatial resolution Remote Sensing Instrument (RSI) is one of the key on-board components. The RSI is a pushbroom scanning type sensor with spectral bands in the visible and near infrared, including panchromatic and four multi-spectral bands (Pan: 520 - $820 \mathrm{~nm}$; Band $1: 450$ - $520 \mathrm{~nm}$; Band $2: 520$ - $600 \mathrm{~nm}$; Band $3: 630$ $690 \mathrm{~nm}$; Band $4: 790$ - $900 \mathrm{~nm}$ ) equipped with 2- and 8-m spatial resolution, respectively. The detectors are aligned in a cross-track direction with a wide field of view over a 24-km swath width. The FORMOSAT-2 satellite orbits the earth at exactly 14 revolutions a day, thus RSI observations can be applied to natural disaster evaluation, agriculture development, land-use and urban planning, environmental monitoring, and ocean surveillance around Taiwan and its neighboring waters.

\footnotetext{
* Corresponding author

E-mail:grliu@csrsr.ncu.edu.tw
}

Before launching a satellite into space, it is very important to accurately calibrate radiometric parameters of the on-board sensor, such as response functions and radiometric conversion coefficients. Preflight calibrations can be conveniently and accurately performed in a laboratory. However, due to different background environments and decay over time, radiometric characteristics of the sensor may still change (Dinguirard and Slater 1999; Hagolle et al. 1999; Barnes et al. 2001). In which case, it is essential not to neglect absolute radiance calibration. In the case of the FORMOSAT-2 RSI, ensuring reliability of radiometric observations is dependent upon the accuracy of absolute observing radiances. This study attempts to develop a viable procedure for retrieving relevant radiometric parameters for the RSI sensor in-orbit.

\section{METHODOLOGY AND FIELD MEASUREMENTS}

The Lunar and Vicarious calibration methods are gener- 
ally used for in-orbit radiometric calibration. The Moon's reflectance is stable enough for calibration, but there are some limitations and uncertainties from the Lunar approach, such as variations in the distance to the Moon (Helfenstein and Veverka 1987; Barnes et al. 1999; Barnes et al. 2001). Alternate calibrations use onboard aircraft instruments to simulate the observed geometry of the satellite overpass. These are just a few of the many different calibration methods that have been proposed and utilized for in-flight radiometric calibration of satellite sensors (Kaufman and Sendra 1988; Staylor 1990; Vermote and Kaufman 1995; Moulin et al. 1996; Eplee et al. 2001). Although several of these methods are indeed practical and accurate, they are also very time-consuming.

Another viable approach is the use of ground-based natural targets. The target sites should be large and flat areas, where reflectance is high and stable (Thome 2001). Based on such a requirement, desert areas appear to be the most suitable choice. Once ground-based equipment is set up at the calibration site, in-orbit calibration can be easily performed during clear and stable atmospheric conditions. Since there are no deserts or desert-like areas on or around Taiwan, various alternate regions have been examined to function as possible calibration sites. Factors considered in calibration-site determination include: atmospheric stability, pollution, cloud cover, and surface reflectance characteristics.

\subsection{Calibration Site Examination}

Suitable calibration sites require: (1) frequent clear atmospheric conditions; (2) minimal seasonal changes; (3) high-spatial uniformity over a large area; and (4) high reflectance and a near lambertian surface. In this study, environmental conditions at potential locations in Taiwan are analyzed for selecting a suitable calibration site for the FORMOSAT-2 RSI. Ground observations from six weather stations of the CWB (Central Weather Bureau, Taiwan) are used for cloud cover analysis during January 2002 to July 2003. Results show that Taipei, Pingtung, Magong, and the Dongsha Island have clearer sky conditions than Hualien and Green Island (see Table 1).

In terms of atmospheric environment, atmospheric aerosol content is considered of major importance due to the visible spectral bands of the RSI. According to the ground measurements of AERONET (Aerosol Robotic Network, Holben et al. 1998), aerosol optical depth over Dongsha Island was almost less than 0.2 in the $440 \mathrm{~nm}$ channel in September 2004, indicating clear skies over Dongsha Island.

In addition to the atmospheric environment, the surface reflective characteristics are also important for any effective radiometric calibration site. The measurements of the ground-based SE590 spectrometer show that reflectance variations in the zenith and azimuth angles over Dongsha Airport (see also Fig. 1) are less than 3.0\% (Fig. 2). Due to its high reflectivity ( $>0.4$, bar RSI band 1 ), and near

Table 1. Analysis of the amount of cloud cover over Hualien, Magong, Pingtung, Green Island, Taipei and Dongsha Island stations during January 2002 to July 2003.

\begin{tabular}{lrrrr}
\hline & \multicolumn{4}{c}{ Cloud Cover } \\
\cline { 2 - 5 } Station & $\mathbf{1 / 8} \sim \mathbf{2 / 8}$ & $\mathbf{3 / 8} \sim \mathbf{4 / 8}$ & $\mathbf{5 / 8} \sim \mathbf{7 / 8}$ & \multicolumn{1}{c}{$\mathbf{8 / 8}$} \\
\hline Hualien & $0 \%$ & $31.73 \%$ & $51.71 \%$ & $16.56 \%$ \\
Magong & $7.67 \%$ & $52.58 \%$ & $35.33 \%$ & $4.41 \%$ \\
Pingtung & $0 \%$ & $68.94 \%$ & $30.16 \%$ & $0.9 \%$ \\
Green Island & $0 \%$ & $38.40 \%$ & $50.54 \%$ & $11.07 \%$ \\
Taipei & $3.23 \%$ & $53.2 \%$ & $43.19 \%$ & $0.36 \%$ \\
Dongsha Island & $7.08 \%$ & $61.99 \%$ & $23.88 \%$ & $7.03 \%$ \\
\hline
\end{tabular}

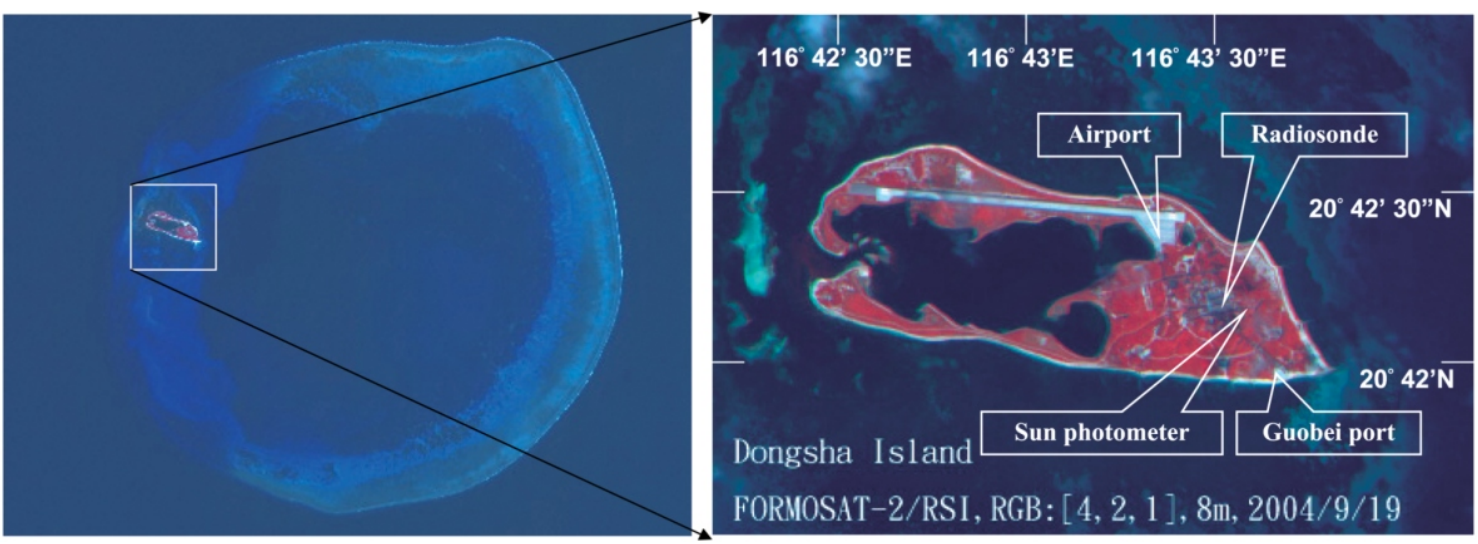

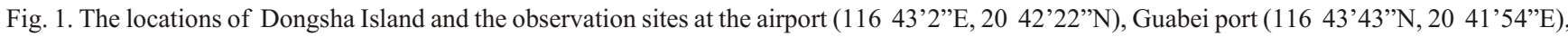

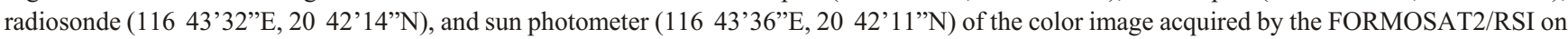
19 September 2004. 
(a)

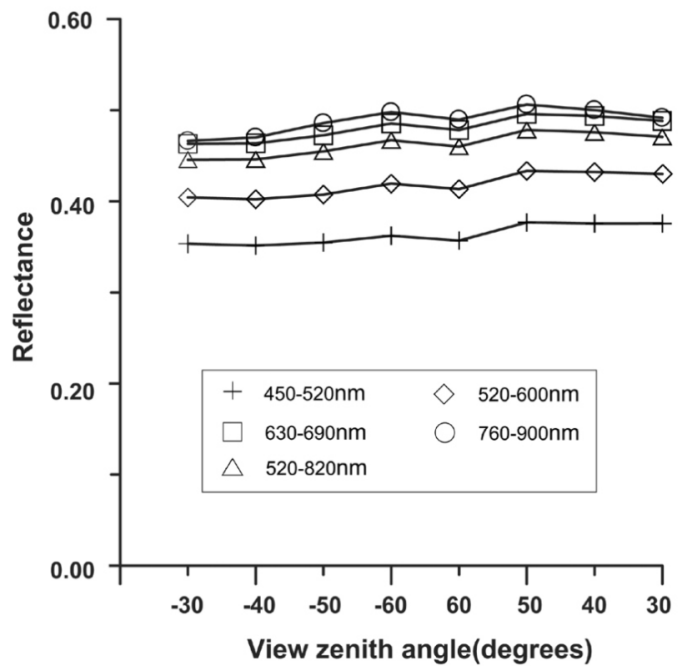

(b)

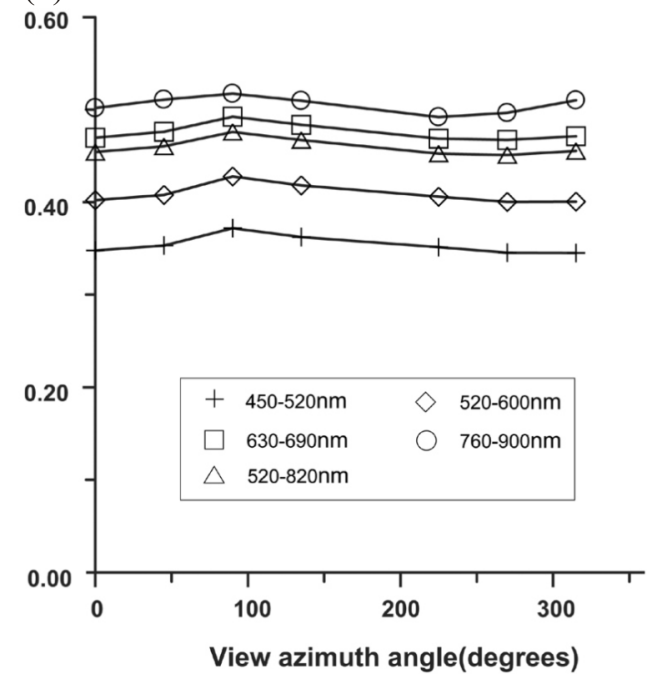

Fig. 2. The surface reflectance of RSI bands at the Dongsha airport measured by the SE590 Spectrometer in terms of different (a) zenith angles and (b) azimuth angles, 17 September 2004.

lambertian surface with a prevalent clear and clean atmospheric environment, the land surface of Donsha airport was considered as an appropriate calibration site for RSI/ FORMOSAT-2 during the first stage.

\subsection{Radiation Transfer of RSI}

Assuming that the ground surface is lambertian, the reflectance observed by the satellite can be expressed as follows (Lin et al. 2002):

$\rho^{*}\left(\mu_{s}, \mu_{v}, \varphi\right)=\rho^{a}\left(\mu_{s}, \mu_{v}, \varphi\right)$

$+\frac{T\left(\mu_{s}\right)\left[\rho \exp \left(-\tau / \mu_{v}\right)+<\rho>t_{d}\left(\mu_{v}\right)\right]}{1-<\rho>s}$

where $\rho^{*}$ is the reflectance observed by the satellite, $\mu_{s}=$ $\cos \theta_{s}, \theta_{s}$ is the solar zenith angle, $\mu_{v}=\cos \theta_{v}, \theta_{v}$ is the sensor zenith angle, $\rho^{a}$ is the atmospheric reflectance, $\phi$ is the relative azimuth angle between the sun and the sensor, $\tau$ is the atmospheric optical depth, $\rho$ is the ground surface reflectance, $T$ is the total transmittance from the sun to the ground surface, $s$ is the atmosphere albedo, $\langle\rho\rangle$ is the mean surface reflectance, and $t_{d}$ is the diffuse transmittance on the surface-sensor path. If the parameters on the right hand side of Eq. (1) can be calculated by the radiative transfer code (LOWTRAN or MODTRAN codes) coupled with ground measurement data (such as sounding data, aerosol properties, and surface reflectance), the satellite-observed reflectance $\left(\rho^{*}\right)$ can be estimated for the in-orbit radiometric calibration. As for the employ of radiative transfer code, a more simplified transmittance/radiation model than LOWTRAN was used to estimate the aerosol optical depth
(AOD) conducted by Holben et al. (1992), and showed a satisfactory result. Therefore, the LOWTRAN-7 model (Kneizys et al. 1988) is used for the multiple scattering and two-way transmittance computations with inputs of radiosonde data and aerosol parameters acquired at Dongsha Island.

In regards to the output data of the RSI, the conversion of the output signal to the spectral radiance is accomplished according to the following relationship:

$C(R)=\left(K_{0} G_{\text {sel }} \eta\right) R+C_{0}+N$

where $C$ is the output signal (digital count) and corresponds to $\rho$ in Eq. (1); $R$ is the mean spectral radiance $\left(W / \mathrm{m}^{2} / \mathrm{sr} /\right.$ $\mu m) ; K_{0}$ is absolute radiometric calibration factor, depending on the band; $G_{s e l}$ is the gain factor attached to the gain number; $\eta$ is the inter-detector equalization coefficient; the gain value, $K_{0} G_{\text {sel }} \eta$, is the conversion factor between digital counts and mean spectral radiance; $C_{0}$ is the dark current for the detector; and $N$ is the noise. In Eq. (2), the gain factor $G_{\text {sel }}$ becomes a constant once the Gain Number (1 $\sim 10$ for RSI sensor) of the RSI sensor is selected, i.e., $G_{\text {sel }}($ GainNumber $)=2^{(\text {GainNumber }-1) / 2}$. Therefore, the absolute radiometric coefficient $\left(K_{0} \eta\right)$ can be calculated with the synchronous dataset of satellite observations $(C)$ and mean spectral radiance derived from field measurements $(R)$, which will be constructed later in the following.

\subsection{The Synchromesh Field Campaign}

In accordance with the results of on-site examinations, an experiment was proposed for the Dongsha airport via synchronous observations from the FORMOSAT-2 satellite 
and ground-based instruments during $16 \sim 20$ September 2004. The on-site instruments include spectroradiometerSE590 and a standard reflection panel for surface reflectance measurements, radiosonde for sounding data observations, and sun photometer-Cimel-318N for aerosol properties detections. Figure 1 indicates the locations of Dongsha Island and the observation sites at the airport $\left(116^{\circ} 43\right.$ '21' $\mathrm{E}$, $\left.20^{\circ} 42^{\prime} 22^{\prime \prime} \mathrm{N}\right)$, Guabei port (116 $\left.46^{\circ} 43^{\prime \prime} \mathrm{N}, 20^{\circ} 41^{\prime} 54^{\prime \prime} \mathrm{E}\right)$, radiosonde $\left(116^{\circ} 43^{\prime} 32^{\prime \prime} \mathrm{E}, 20^{\circ} 42^{\prime} 14^{\prime \prime} \mathrm{N}\right)$ and sun photometer $\left(116^{\circ} 43^{\prime} 36^{\prime \prime} \mathrm{E}, 20^{\circ} 42^{\prime} 11^{\prime \prime} \mathrm{N}\right)$ based on a color image acquired by FORMOSAT2/RSI on 19 September 2004. The procedure for RSI absolute radiance calibration with synchromesh field measurements is shown in Fig. 3, which includes sounding data, surface reflectivity for different zenith and azimuth angles (spectroradiometer), and the aerosol optical depth (sun photometer). Afterwards, the measurements of the synchronous experiment were applied to the radiometric calibration of the in-orbit RSI sensor.

From the on-site measurements of sounding data, aerosol properties, and the surface reflectance (Dongsha airport), the mean spectral radiance of RSI bands can be derived by using LOWTRAN-7 code coupled with the FORMOSAT-2 viewing and solar geometry. And then, the GAIN values $\left(K_{0} G_{\text {sel }} \eta\right)$ of RSI spectral bands can be obtained from the mean spectral radiance $(R)$ and the correspondent Digital Counts of SRI observed $[C(R)]$ through Eq. (2). As a result, the absolute radiometric calibration factors $\left(K_{0} \eta\right)$ can be constructed for in-orbit FORMOSAT-2 RSI.

\section{RESULTS AND ANALYSIS}

There are five datasets acquired during the period of the synchronous field experiment, 16 20 September 2004. Due to cloud effect during the experiment, only the data observed on September 17 and 19 in 2004 were used for the RSI radiometric calibration. Radiometry parameters for the RSI sensor in-orbit were obtained via measurements from the FORMOSAT-2 satellite and ground-based instruments with the radiance transfer models. The results of the retrieved GAIN values of RSI bands are shown in Table 2. From the GAIN values in Table 2, two types of gain numbers are obviously used for the observation mode on September 17 and 19, respectively. As for the RSI sensor, the absolute radiometric calibration factor, $K_{0} \eta$, in each band should be a constant value no matter what gain number used, i.e., $G_{\text {sel }}$. Therefore, the cases operated in different observation modes (gain number 3 and 4 ) provide a good opportunity to validate the results of absolute radiometric calibration in this study. Table 3 illustrates the absolute radiometric calibration factor $\left(K_{0} \eta\right)$ of the RSI bands derived in the two gain number modes. The difference of $K_{0} \eta$ between the two modes in each RSI band is less than $5 \%$ - an indication of high consistency and practicability of the proposed procedure in this study.

Furthermore, the results of retrieved surface reflectance are validated with the ground measurements at the location of Guobei port (116 $43^{\circ} 43^{\prime \prime}$, 20 2041’54”E, see also Fig. 1).

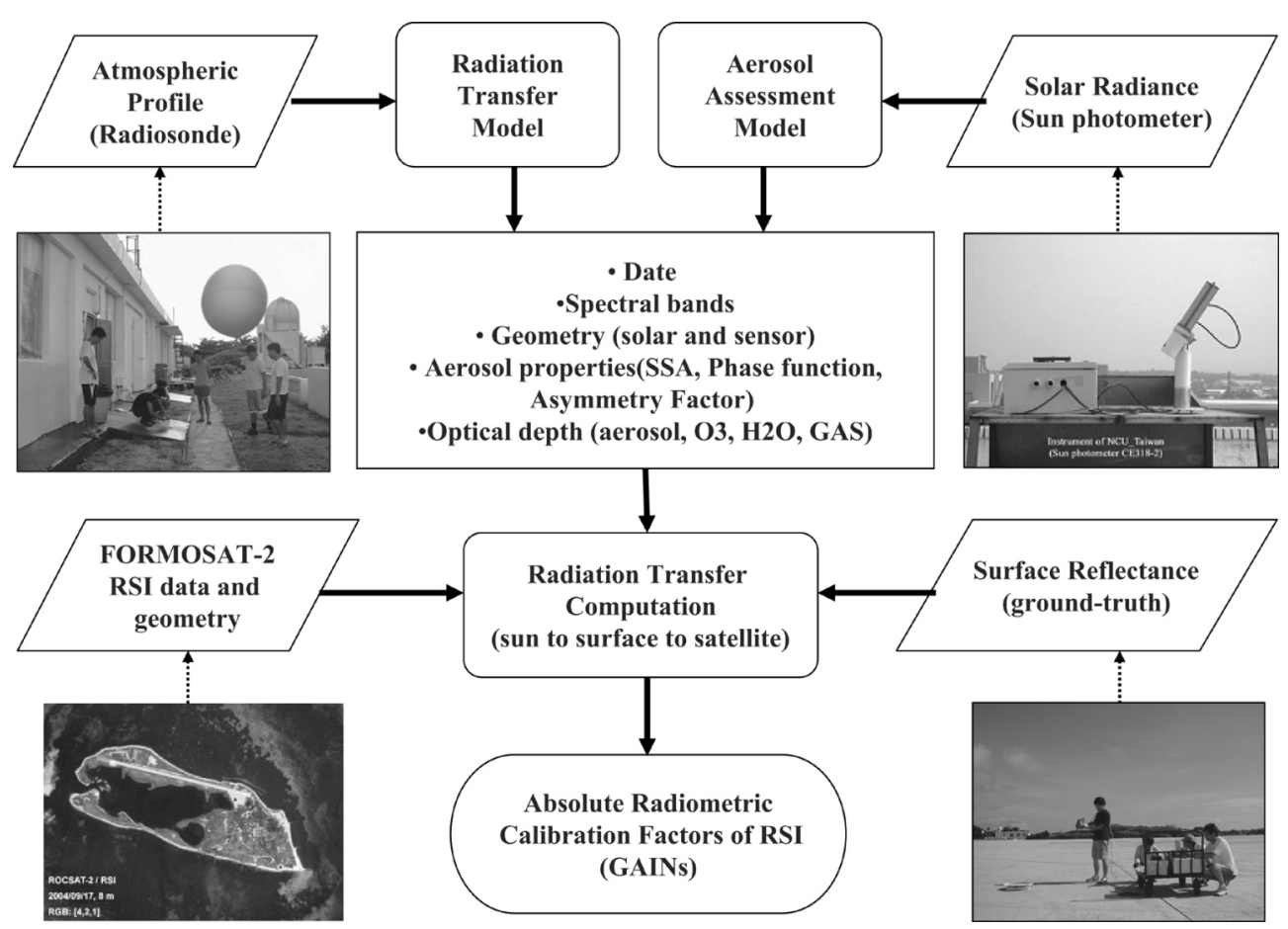

Fig. 3. The flow chart of the in-orbit FORMOSAT-2 RSI absolute radiometric calibration. 
Table 2. The derived GAIN values of the in-orbit RSI sensor.

\begin{tabular}{ccc}
\hline \multicolumn{3}{c}{ GAIN values of RSI sensor $\left(\boldsymbol{K}_{\mathbf{0}} \boldsymbol{G}_{\text {sel }} \boldsymbol{\eta}\right)$} \\
\hline RSI band & Gain Number $=\mathbf{3}(\mathbf{2 0 0 4 . 0 9 . 1 7})$ & Gain Number $=\mathbf{4}$ (2004.09.19) \\
\hline Band $1(450-520 \mathrm{~nm})$ & 0.8499 & 1.1770 \\
Band $2(520-600 \mathrm{~nm})$ & 0.7907 & 1.0678 \\
Band 3(630 $-690 \mathrm{~nm})$ & 0.7757 & 1.0616 \\
Band 4(760 - $900 \mathrm{~nm})$ & 1.1086 & 1.6023 \\
Pan $(520-820 \mathrm{~nm})$ & 0.9211 & 1.3118 \\
\hline Radiance $\left(\mathrm{W} / \mathrm{m}^{2} / \mu \mathrm{m} / \mathrm{sr}\right)=$ Digital Counts / GAIN value \\
\hline
\end{tabular}

Table 3. The derived absolute radiometric calibration factors of the in-orbit RSI sensor.

\begin{tabular}{cccc}
\hline \multicolumn{4}{c}{ Absolute radiometric calibration factors of RSI sensor $\left(\boldsymbol{K}_{\mathbf{0}} \boldsymbol{\eta}\right)$} \\
\hline RSI band & Gain Number $=\mathbf{3}(\mathbf{2 0 0 4 . 0 9 . 1 7 )}$ & Gain Number $=\mathbf{4}(\mathbf{2 0 0 4 . 0 9 . 1 9 )}$ & Difference \\
\hline Band 1 & 0.4250 & 0.4161 & $2.1 \%$ \\
Band 2 & 0.3954 & 0.3775 & $4.5 \%$ \\
Band 3 & 0.3879 & 0.3753 & $3.2 \%$ \\
Band 4 & 0.5543 & 0.5665 & $2.2 \%$ \\
Pan & 0.4606 & 0.4638 & $0.7 \%$ \\
\hline
\end{tabular}

The surface reflectance of Guobei port derived with the absolute radiometric conversion (GAIN values in Table 2) and radiative transfer code coupled with ground measurement data (sounding data and aerosol properties) illustrates satisfactory results when compared with the ground-based measurements of the spectroradiometer-SE590 (less than 5\%), as shown in Table 4. In other words, the method (procedure) pursued in this study appears to be feasible, and it can be successfully applied to the absolute radiometric calibration of the RSI sensor. Since the RSI sensor has 10 gain numbers designed for a larger radiometric dynamic range in the applications of both low and high reflectance objects, correspondence in the GAIN values (the coefficients of absolute radiometric conversion) to each respective gain number of the FORMOSAT2/RSI was also constructed (Fig. 4).

\section{DISCUSSIONS}

The procedure and radiometry parameters for the absolute radiometric calibration of the in-orbit FORMOSAT-2 RSI sensor were successfully constructed by a field campaign between synchronous satellite and ground-based observations. The results not only reveal the absolute radiometric conversion of the RSI data for scientific research, but also delineated the radiometric calibration procedure for the
Table 4. Comparison of the ground measurements with the RSI bands' calculated reflectance at the Guobei port.

\section{Surface Reflectivity of RSI Bands at Guobei}

Band 1 Band 2 Band 3 Band 4 Pan

\begin{tabular}{llllll} 
2004.09.17 & & & & & \\
Measured & 0.42 & 0.47 & 0.50 & 0.51 & 0.48 \\
Retrieved & 0.40 & 0.46 & 0.49 & 0.49 & 0.46 \\
Error(\%) & 4.7 & 2.1 & 2.0 & 3.9 & 4.1 \\
2004.09.19 & & & & & \\
Measured & 0.44 & 0.49 & 0.52 & 0.53 & 0.52 \\
Retrieved & 0.43 & 0.51 & 0.53 & 0.51 & 0.50 \\
Error(\%) & 2.2 & 4.0 & 1.9 & 3.8 & 4.0 \\
\hline
\end{tabular}

RSI data. Maintaining the quality of the RSI data is extremely important for the mission of FORMOSAT-2. Therefore, periodical experiments to attain absolute radiometric calibration should be continuously performed to ensure the quality of RSI data.

Acknowledgements This research was primarily supported by the National Space Organization (grant No.: 93- 


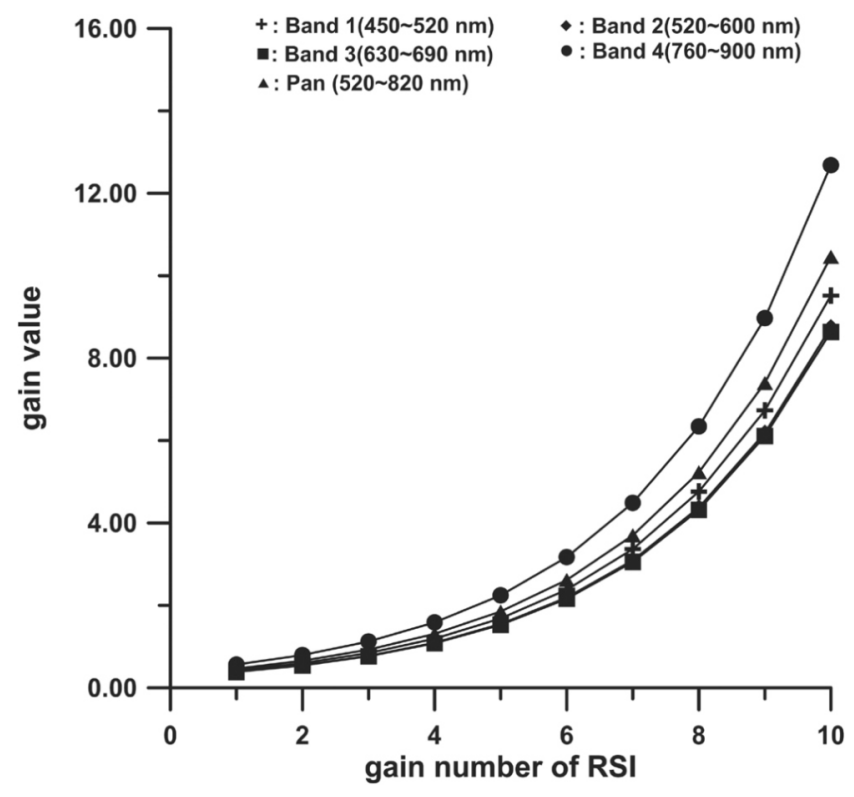

Fig. 4. The GAIN values correspond to each gain number $(1 \sim 10)$ of the FORMOSAT2/RSI spectral bands.

NSPO(B)-RGS-FA07-02) and the National Science Council (grant No.: NSC 96-2752-M-008-005 -PAE) of Taiwan. The authors would like to thank Dr. D. Y. Liu and Dr. N. Y. Chen of NSPO, and Mr. W. W. Lin of CSRSR NCU, in offering their full support during the Dongsha Island experiment. Our thanks are also extended to Mr. Charlie Liang for his kindness in reviewing our written English.

\section{REFERENCES}

Barnes, R. A., R. E. Eplee Jr., F. S. Patt, and C. R. McClain, 1999: Changes in the radiometric sensitivity of SeaWiFS determined from lunar and solar-based measurements. Appl. Optics, 38, 4649-4664, doi: 10.1364/AO.38.004649. [Link]

Barnes, R. A., R. E. Eplee, G. M. Schmidt, F. S. Patt, and C. R. McClain, 2001: Calibration of SeaWiFS. I. Direct techniques. Appl. Optics, 40, 6682-6700, doi: 10.1364/AO.40. 006682. [Link]

Dinguirard, M. and P. N. Slater, 1999: Calibration of spacemultispectral imaging sensors: A review. Remote Sens. Environ., 68, 194-205, doi: 10.1016/S0034-4257(98)001114. [Link]

Eplee, R. E., W. D. Robinson, S. W. Bailey, D. K. Clark, P. J. Werdell, M. Wang, R. A. Barnes, and C. R. McClain, 2001: Calibration of SeaWiFS. II. Vicarious techniques. Appl. Optics, 40, 6701-6718, doi: 10.1364/AO.40.006701. [Link]
Hagolle, O., P. Goloub, P. Y. Deschamps, H. Cosnefroy, X. Briottet, T. Bailleul, J. M. Nicolas, F. Parol, B. Lafrance, and M. Herman, 1999: Results of POLDER in-flight calibration. IEEE Trans. Geosci. Remote Sensing, 37, 15501566, doi: 10.1109/36.763266. [Link]

Helfenstein, P. and J. Veverka, 1987: Photometric properties of lunar terrains derived from Hapke's equations. Icarus, 72, 342-357, doi: 10.1016/0019-1035(87)90179-5. [Link]

Holben, B. N., E. Vermot, Y. J. Kaufman, D. Tanre, and V. Kalb, 1992: Aerosol retrieval over land from AVHRR data-application for atmospheric correction. IEEE Trans. Geosci. Remote Sensing, 30, 212-222, doi: 10.1109/36.134072. [Link]

Holben, B. N., T. F. Eck, I. Slutsker, D. Tanré, J. P. Buis, A. Setzer, E. Vermote, J. A. Reagan, Y. J. Kaufman, T. Nakajima, F. Lavenu, I. Jankowiak, and A. Smirnov, 1998: GAERONET-A federated instrument network and data archive for aerosol characterization. Remote Sens. Environ., 66, 1-16, doi: 10.1016/S0034-4257(98)00031-5. [Link]

Kaufman, Y. J. and C. Sendra, 1988: Algorithm for automatic atmospheric corrections to visible and near-IR satellite imagery. Int. J. Remote Sens., 9, 1357-1381, doi: 10.1080/ 01431168808954942. [Link]

Kneizys, F. X., E. P. Shettle, L. W. Abreu, J. H. Chetwynd, G. P. Anderson, W. O. Gallery, J. E. A. Selby, and S. A. Clough, 1988: Users guide to LOWTRAN 7, Report AFGL-TR88-0177, Air Force Cambridge Research Laboratories, Bedford, MA, August, $137 \mathrm{pp}$.

Lin, T. H., A. J. Chen, G. R. Liu, and T. H. Kuo, 2002: Monitoring the atmospheric aerosol optical depth with SPOT data in complex terrain. Int. J. Remote Sens., 23, 647-659, doi: 10.1080/01431160110069827. [Link]

Moulin, C., C. E. Lambert, J. Poitou, and F. Dulac, 1996: Long term (1983-1994) calibration of the Meteosat solar (VIS) channel using desert and ocean targets. Int. J. Remote Sens., 17, 1183-1200, doi: 10.1080/01431169608949076. [Link]

Staylor, W. F., 1990: Degradation rates of the AVHRR visible channel for the NOAA 6, 7 and 9 spacecraft. J. Atmos. Ocean. Technol., 7, 411-423, doi: 10.1175/1520-0426 (1990)007<0411:DROTAV>2.0.CO;2. [Link]

Thome, K. J., 2001: Absolute radiometric calibration of Landsat $7 \mathrm{ETM}+$ using the reflectance-based method. Remote Sens. Environ., 78, 27-38, doi: 10.1016/S0034-4257(01) 00247-4. [Link]

Vermote, E. and Y. J. Kaufman, 1995: Absolute calibration of AVHRR visible and near infrared using ocean and cloud views. Int. J. Remote Sens., 16, 2317-2340, doi: 10.1080/ 01431169508954561. [Link] 